\title{
Viviendo en la incertidumbre después de un macroadenoma pituitario: un caso de estudio
}

\author{
Redondo-Sáenz, Diego'; Solano-López, Ana Laura
}

\section{RESUMEN}

Introducción: La Teoría de la Incertidumbre frente a la Enfermedad es una teoría de enfermería intermedia que explica los procesos que los individuos con enfermedades crónicas atraviesan con el objetivo de lograr la adaptación. Esta teoría describe antecedentes de la incertidumbre, mecanismos de valoración y estrategias de afrontamiento para adaptarse a nuevas condiciones. Objetivo: Demostrar la integración del proceso de enfermería, lenguaje estandarizado y la Teoría de la Incertidumbre en la Enfermedad en el cuidado de una persona con complicaciones físicas y psicosociales tras un macroadenoma hipofisiario. Metodología: Los datos fueron recolectados a través de entrevistas con el paciente, examen físico y el expediente médico. La Teoría de la Incertidumbre frente a la Enfermedad de Mishel, lenguajes estandarizado para describir los diagnósticos, intervenciones y resultados y evidencia científica fueron utilizados para desarrollar el plan de cuidados. Presentación del caso: La teoría de la incertidumbre permitió seleccionar el diagnóstico principal (Afrontamiento ineficaz relacionado con incertidumbre), el cual se abordó con los NIC: mejorar el afrontamiento y enseñanza: proceso de la enfermedad, los resultados medidos fueron NOC: nivel de estrés y conocimiento: manejo de la enfermedad crónica. La efectividad de este enfoque fue reflejado en la mejora de los indicadores. Conclusiones. La identificación de una teoría que pueda explicar los hallazgos y guiar las intervenciones fortalece la calidad del cuidado de enfermería. Esta integración facilita un cuidado de enfermería personalizado y basado en la evidencia.

Palabras clave: Incertidumbre; Enfermedad Crónica; Proceso de Enfermería (DeCS; BIREME)

'Enfermero, San José, Costa Rica. ORCID ID: 0000-0001-9321-8719

2Profesora, Escuela de Enfermería, Universidad de Costa Rica, San José, Costa Rica. Ciudad de la Investigación, Montes de Oca, San José, Costa Rica. Email: analaura.solanolopez@ucr.ac.cr. ORCID-ID: 0000-0002-0151-3751

Recibido: $11 / 02 / 2019$

Aceptado: 09/05/2019

*Autor para correspondencia

\section{Cómo citar este artículo}

Redondo-Sáenz D, Solano-López AL. Viviendo en la incertidumbre después de un macroadenoma pituitario: un caso de estudio. SANUS. 2019; (10): 85-99. [Acceso_- $\frac{\text { ] }}{\text {; }}$; Disponible en: mes día año URL 


\title{
Deixando de incerteza após uma macroadenoma pituitária: un estu- do de caso
}

\section{RESUMO}

\begin{abstract}
Introdução: A Teoria da Incerteza na Doença é uma teoria intermediária de enfermagem que explica os processos enfrentados por pessoas com doenças crônicas, a fim de alcançar a adaptação. Objetivo: Demonstrar a integração do processo de enfermagem, linguagem padronizado, e a teoria da incerteza na doença envolvida no cuidado de uma pessoa com complicações físicas e psicossociais após um macroadenoma hipofisário. Metodologia: Os dados foram coletados por meio de entrevistas com o paciente, exame físico e registro médico. Teoria da incerteza na doença de Mishel, linguagem padronizado para descrever o diagnóstico, intervenções, resultados e evidências cientificas foram utilizados para desenvolver o plano de cuidados. Apresentação do caso: A Teoria da Incerteza permitiu selecionar o diagnóstico principal (enfrentamento ineficaz relacionado à incerteza), que foi abordado com o NIC: melhorar o enfrentamento e a aprendizagem, os resultados medidos foram NOC: nível de estresse e conhecimento: gestão de doenças crônicas. A eficácia desta abordagem reflectiu-se na melhoria dos indicadores. Conclusões: A identificação de uma teoria que possa explicar os achados e orientar as intervenções fortalece a qualidade do cuidado de enfermagem. Esta integração facilita um atendimento de enfermagem personalizado e baseado em evidências.
\end{abstract}

Palavras chave: Incerteza; doença crônica; processo de enfermagem (DeCS; BIREME).

\section{INTRODUCCIÓN}

La Teoría de la Incertidumbre frente a la Enfermedad explica cómo las personas abordan la incertidumbre, definida como la incapacidad de determinar el significado de los eventos relacionados con la enfermedad ${ }^{(1,2)}$. La incertidumbre, entendida más como un proceso que como un estado estático, se encuentra precedida por el marco de los estímulos, las capacidades cognitivas y los proveedores de estructura $a^{(1)}$. Una vez presentada, sufre un proceso de valoración por medio de los mecanismos cognitivos de la inferencia y la ilusión, con los resultados probables de la valoración como peligro y oportunidad(1). En caso de que la persona transcurra con la incertidumbre por largos períodos y sus antecedentes sean lo suficientemente consistentes para promover el cambio, la valoración cambia hacia una nueva perspectiva de vida regida por un pensamiento probabilístico(2). Por lo tanto, esta teoría sirve de guía para entender los procesos de salud y enfermedad y así proveer cuidados de enfermería adecuados para ayudar las personas a manejar la incertidumbre.

En este caso, el Sr. R. fue súbitamente diagnosticado con un macroadenoma pituitario. Esta entidad patológica consiste en un tumor generalmente benigno en la glándula hipófisis, pudiendo ser de naturaleza secretora o no, cuyo tratamiento de primera línea consiste en resección quirúrgica de abordaje trans-esfenoidal endonasal mínimamente invasivo(3). Se estima una incidencia de 12 a 42 casos por millón de habitantes por año, y tras la resección, el 52,3\% de las personas reportan al menos una deficiencia pituitaria, siendo las más comunes aquellas deficiencias relacionadas con el eje hipotálamo-hipófisis-glándula periférica ${ }^{(4)}$. En concordancia con esto, como resultado del adenoma y de la resección quirúrgica, el Sr. R. desarrolló múltiples condiciones crónicas que requirieron tratamiento continuo y adaptación. Es en este último proceso, relativo al período post-quirúrgico, donde usualmente se presenta un mayor impacto en la vida de las personas con un tumor hipofisiario, con la particularidad de que el impacto no es solo debido a las consecuencias físicas de la enfermedad y el tratamiento sino también al impacto en los dominios psicológico y social de los mismos ${ }^{(5)}$. Más aún, es en los síntomas del período post-quirúrgico (problemas de visión, de la función sexual y cambios hormonales, entre otros) donde se encuentra la génesis de la baja calidad de vida de estos pacientes6. En concordancia con lo anterior, el Sr. R. mostró algunas 
dificultades para entender y manejar su nueva situación social y de salud, experimentando incertidumbre. Por lo tanto, el objetivo de este artículo es demostrar la integración del proceso de enfermería, lenguaje estandarizado y la Teoría de la Incertidumbre en la Enfermedad en un plan de cuidados para apoyar a la persona en sus complicaciones físicas y psicosociales.

\section{METODOLOGÍA}

Para la gestión del cuidado del Sr. R se utilizó el proceso de enfermería, el cual es un proceso sistemático y científico para identificar y resolver problemas de salud así como reconocer factores protectores para favorecerlos y así aumentar el bienestar; este consta de 5 etapas: valoración, diagnóstico, planeamiento, ejecución y evaluaciónn ${ }^{(7)}$. La fase de valoración focalizada estuvo guiada por los patrones funcionales de Marjory Gordon ${ }^{(8)}$. Una vez encontradas las respuestas y necesidades humanas se elaboró un plan de cuidados utilizado las taxonomía-Il de la NANDAInternacional9 para los diagnósticos, $\mathrm{NOC}^{(10)}$ para el establecimiento de resultados e indicadores y $\mathrm{NIC}^{(11)}$ para seleccionar las intervenciones y acciones más apropiadas según el caso y el contexto. El proceso se llevó a cabo en el curso de tres días de la misma semana, los cuales correspondían a la práctica clínica del curso EE0421 Módulo 5: Intervención de Enfermería con la Adultez en Procesos Mórbidos ${ }^{(11)}$. Durante todo el proceso se utilizó como guía la teoría de la Incertidumbre en la Enfermedad.

\section{PRESENTACIÓN DEL CASO}

\section{Valoración}

El Sr. R. es un hombre de 44 años, que no completó la educación secundaria y trabajaba como conductor de camión. Actualmente se encontraba desempleado.

A continuación se detallan los hallazgos de la valoración por medio de la revisión del expediente clínico, el examen físico y el historial de enfermería (entrevista), los cuales son presentados según los Patrones Funcionales de Gordon ${ }^{(8)}$.

Patrón Percepción -Manejo de la Salud: En el 2015, el Sr. R. sufrió una neuritis óptica bilateral causada por un macroadenoma pituitario. El tratamiento para esta condición fue una resección trans-esfenoidal. Como resultado del procedimiento, el nervio óptico fue irremediablemente dañado y el eje hipotálamo-hipófisis interrumpido. Por lo tanto, desarrolló hipotiroidismo secundario, hipogonadismo, anosmia y ceguera. El Sr. R. fue admitido a la unidad de neurociencias debido a una meningitis bacteriana, causada por un defecto en el hueso esfenoides y también presentaba anemia hipercrómica. Se encontraba bajo tratamiento farmacológico: 1) Enalapril 20mg vía oral cada día, 2) Levotiroxina 1 tableta vía oral cada día, 3) Fludrocortisona 1 tableta vía oral cada día, 4) Cefotaxime $2 \mathrm{~g}$ intravenoso cada 8 horas, 5) Vancomicina $1 \mathrm{~g}$ intravenoso cada 8 horas. Los exámenes de laboratorio tenían los siguientes valores:

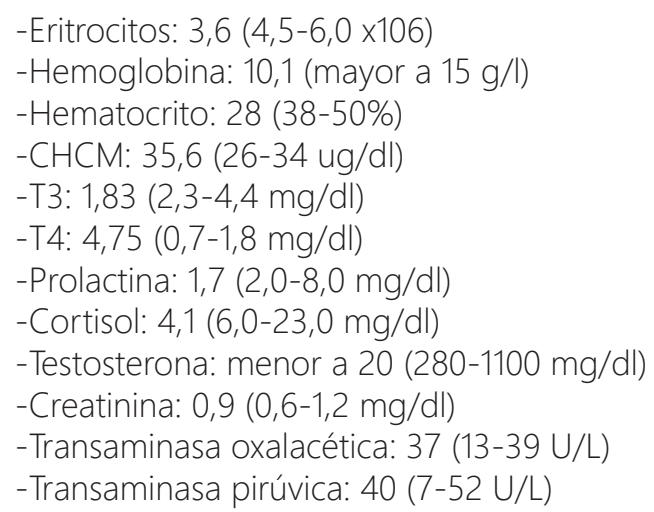

Previo al macroadenoma el Sr. R. manifiesta haber consumido alcohol de manera esporádica, pero al momento del diagnóstico detuvo su consumo. Como antecedente patológico personal tiene hipertensión y no tiene antecedentes quirúrgicos ni alergias. Sus signos vitales se identificaron como estables, a saber: presión arterial: 109/73 mmHg; frecuencia cardíaca: 96 latidos por minuto; frecuencia respiratoria: 16 respiraciones por minuto y temperatura: $36,5^{\circ} \mathrm{C}$. Él estaba a la espera del egreso cuando se proveyó la atención de enfermería.

Patrón Nutricional-Metabólico: El peso y la talla no se encontraban disponibles al momento de la valoración, ni tampoco se contaba en la unidad con los elementos para tomarlos. El Sr. R. manifestó consumir una dieta basada en cereales, con carne de pollo, res o cerdo; con poco pescado y alta cantidad de verduras y vegetales (principalmente consumidos hervidos o en sopa). Menciona consumir "poca" agua durante el día, si bien no es capaz de precisar la cantidad. La baja hemoglobina y hematocrito y una aumentada concentración de hemoglobina corpuscular media indica el estado de anemia hipercrómica secundaria al proceso infeccioso constatada en el expediente clínico.

Patrón Eliminación: El Sr. R. reportó mantener un patrón de defecación normal, sin dificultad, defecando al menos una vez al día. Micciona frecuentemente durante el día, sin precisar cantidad exacta ni características de la misma, y sin dificultades a la hora de la micción.

Patrón Actividad-Ejercicio: El Sr. R. tenía dificultades con las actividades básicas de la vida diaria (como comer y caminar) y actividades instrumentales de la vida diaria (como tomar su medicación). Se observó marcha inestable y uso de ayudas biomecánicas (bastón). Menciona que por el reciente diagnóstico tuvo que interrumpir su patrón de actividad física, otrora provechoso, debido principalmente a la falta de acostumbramiento a su nueva condición. A pesar de la anemia hipercrómica que presenta, este 
síndrome no tiene efectos evidentes en su tolerancia a la actividad. Durante el examen físico se constató un choque de punta a nivel del quinto espacio intercostal izquierdo y la línea medioclavicular, lo cual resulta relevante a la luz de su diagnóstico de hipertensión arterial de larga data, pues sugiere la ausencia de una cardiomegalia. La auscultación cardiopulmonar no reveló alteraciones.

Patrón Sueño-Descanso: El Sr. R. menciona poder conciliar el sueño fácilmente, describiéndolo como reparador y estimando un aproximado de 7 horas de sueño en promedio.

Patrón Cognitivo-Perceptivo: El Sr. R. mencionó su "incapacidad" para involucrarse en su autocuidado debido a su falta de conocimiento sobre sus condiciones crónicas derivadas de su condición clínica previa, complicaciones y tratamientos, concretamente refirió vacíos de información referentes a la ceguera y el hipotiroidismo. Estos hallazgos se contrastan con los obtenidos durante el examen físico: la presencia de pupilas midriáticas sin reacción a la luz y la incapacidad de discriminar olores corroboran la lesión de los nervios olfatorio, óptico y oculomotor por la neuritis óptica, la presión del macroadenoma sobre el quiasma óptico y el abordaje transesfenoidal, lo que, a su vez, da cuenta del déficit sensitivo de la ceguera y la anosmia.

Patrón Autopercepción-Autoconcepto: El Sr. R. expresó sentimientos de ansiedad y miedo. No obstante, como éstos no repercutían en su autopercepción, siguiendo su génesis son descritos en los patrones Rol-Relaciones y Afrontamiento-Tolerancia al estrés.

Patrón Rol-Relaciones: El Sr. R. estaba casado y tenía tres hijos. Convive en su casa alquilada con su esposa y dos de sus hijos. Dudaba al tomar decisiones en su vida, especialmente aquellas relacionadas con su empleo, ya que su anterior empleo como conductor de camión se ve imposibilitado por su actual condición de ceguera. El desempleo agravaba la situación, pues el único apoyo financiero de su hogar era su hijo mayor. Por otra parte, fue enfático en su conflicto con el rol de proveedor mediante verbalizaciones como la siguiente: "me da miedo no poder mantener a mis hijos". Más aún, su esposa era la cuidadora principal, lo que lo hacía sentirse ansioso porque no quería convertirse totalmente dependiente de ella. Enfatiza a la vez el apoyo de su esposa, sus amigos y comunidad como su mayor sostén para enfrentar la "situación inmanejable". Sus vecinos, los considera amigos, y han estado cercanos al proceso de enfermedad.

Patrón Sexualidad-Reproducción: El Sr. R. refirió tener problemas en sus relaciones sexuales concernientes a la incapacidad de mantener una excitación constante durante el coito. A la vez, se observaron signos de hipogonadismo durante el examen físico, contrastados con el nivel extremadamente bajo de testosterona sérica. A pesar de esta problemática, el participante declinó proveer mayor información a este respecto y manifestó que no deseaba que este problema fuera abordado.

Patrón Afrontamiento-Tolerancia al estrés: El Sr. R. reportó tener dificultades al adaptarse a la nueva situación, específicamente a la ceguera y el amplio tratamiento médico. Menciona que debido a su desempleo se dedica a "estar en su casa sin hacer nada" y que se dedica a aprender a adaptarse a su ceguera. Cuando se le preguntó acerca de su afrontamiento a la situación, enfatizó en dos sentimientos: miedo y ansiedad. Le asustaban los grandes cambios en su vida y el futuro. También, sentía nostalgia por el pasado y experimentaba altos niveles de estrés, por lo que evitaba las situaciones que le provocaban estrés, ansiedad o nostalgia. Además menciona que una de sus mayores preocupaciones es la limitación económica, ya que el único aporte económico es su hijo mayor, por lo tanto se le está tramitando una pensión por invalidez.

Patrón Valores-Creencias: El Sr. R. mencionó que su religión era católica, sin embargo, apuntó que la misma no juega un papel fundamental en su vida y se declara "no practicante".

\section{Diagnóstico}

Tras el análisis de los datos de la valoración, se determinaron las respuestas humanas en la forma de diagnósticos de enfermería. Se utilizaron los lenguajes enfermeros estandarizados de NANDA-Internacional Taxonomía $\| 9$, la Clasificación de Resultados de Enfermería (NOC)(10) y la Clasificación de Intervenciones de Enfermería (NIC) ${ }^{(11)}$; para proveer cuidado enfermero personalizado. Se consideraron múltiples diagnósticos y se utilizó el modelo AREA (Figura 1) para asegurar un razonamiento clínico apropiado y pensamiento crítico con el fin de priorizar los diagnósticos ${ }^{(13)}$. Afrontamiento ineficaz relacionado con incertidumbre fue seleccionado como diagnóstico prioritario debido a que la incertidumbre valorada como peligro no está permitiendo que el Sr. R. desarrolle estrategias de afrontamiento efectivas ${ }^{(1)}$. Conocimientos deficientes relacionado con información insuficiente fue seleccionado como diagnóstico secundario porque un adecuado conocimiento acerca de las enfermedades, el tratamiento y las consecuencias puede proveer un esquema cognitivo para procesar la situación y ayudar al desarrollo de mecanismos de afrontamiento(1). Estos dos diagnósticos tienen un efecto directo en el diagnóstico Conflicto de decisiones.

Los otros diagnósticos relacionados con actividad/ descanso y sexualidad fueron descartados, pues si se proveía adecuado apoyo para el desarrollo de estrategias de afrontamiento e información, el Sr. R. podría mejorar su valoración de los estresores, seleccionar respuestas adecuadas y utilizar eficazmente los recursos disponibles ${ }^{(14)}$, como el apoyo familiar y el sistema de salud. 
Figura 1. Modelo AREA para la priorización del diagnóstico

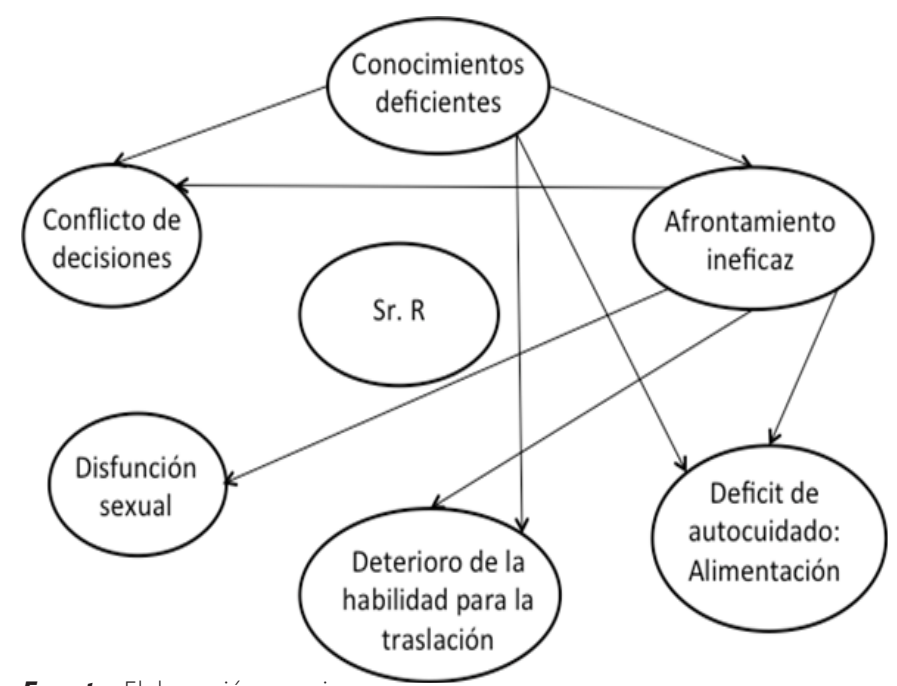

Fuente: Elaboración propia

\section{Planeamiento y Ejecución}

En concordancia con los diagnósticos de enfermería, se utilizó el lenguaje taxonómico mencionado para proveer cuidado personalizado. El plan de cuidados completo es resumido en la Tabla 1.

Respecto al diagnóstico primario, el Sr. R. aceptó seleccionar Nivel de Estrés como el resultado principal. Las personas con altos niveles de incertidumbre, especialmente en condiciones donde la incertidumbre es valorada como peligro, tienden a tener altos niveles de estrés y ansiedad ${ }^{(14)}$. En este caso, el Sr. R. valoraba la incertidumbre como peligro y experimentaba altos niveles de estrés y ansiedad, lo cual es concordante con varios estudios ${ }^{(15,16)}$. Por tanto, una intervención enfocada en disminuir los niveles de estrés como resultado es válida en esta situación. Por otra parte, Conocimiento: Manejo de la enfermedad crónica fue seleccionado como el resultado para el diagnóstico secundario. Amparado en la teoría de Mishel, el objetivo fue proveer estructura con tal de mejorar el marco de los estímulos e, indirectamente, disminuir el nivel de incertidumbre ${ }^{(17)}$.

El estrés fue considerado un signo primario de la incertidumbre18 valorada como peligro, consecuentemente, se seleccionó un NIC11 que apoyara los esfuerzos de afrontamiento en una intervención a largo plazo. Por tanto, Mejorar el afrontamiento se seleccionó como intervención (tabla 1). Esta fue elegida porque podría ser implementada en el hospital y la comunidad, permitiendo la continuidad de los cuidados entre el hospital y los centros de atención primaria.

A la vez, la falta de conocimiento acerca de las condiciones, los tratamientos y sus potenciales complicaciones podrían estar contribuyendo en la percepción de la incertidumbre como peligro. Por tanto, se seleccionó la intervención Enseñanza: Proceso de la enfermedad. Cabe destacar que así como el diagnóstico de Conocimientos deficientes fue seleccionado como secundario, esta intervención también lo fue, toda vez que proveer información sin trabajar antes con la incertidumbre puede resultar contraproducente en los niveles de estrés y ansiedad19.

\section{Evaluación}

Como puede notarse en el plan de cuidados (tabla 1)., el nivel de estrés observado el día después de la intervención fue menor, dado que el Sr. R. mencionó que expresar sus sentimientos: "sacó lo que tenía dentro". Más aún, todos los indicadores de conocimiento alcanzaron la puntuación más alta, debido a que cuando se le hicieron preguntas específicas acerca de los contenidos de la sesión educativa, demostró una adecuada comprensión de sus condiciones y su tratamiento. Por tanto, la intervención fue eficaz al mejorar el conocimiento acerca de sus condiciones crónicas. Sin embargo, es de notar que una vez egresado, requerirá más apoyo educativo, a la vez que es necesario proporcionar un cuidado de enfermería que incluya no solamente al paciente sino también a su pareja, objeto de estudio de las últimas investigaciones relacionadas con la incertidumbre ${ }^{(20)}$. El comprender a su pareja no solamente como parte de su familia sino también como una persona que eventualmente experimentará incertidumbre como fruto de sus acciones de cuidado hacia su esposo(21) permitiría indirectamente apoyarle a él incentivando el apoyo social.

\section{DISCUSIÓN}

Durante la fase de valoración, se identificaron muchas fuentes de incertidumbre. De acuerdo con la teoría, un antecedente de incertidumbre es el marco de los estímulos, compuesto por tres dimensiones: patrón de síntomas, familiaridad con el evento y congruencia del evento1. En el caso del Sr. R, todas las condiciones crónicas que experimentó tenían una consistencia a lo largo del tiempo y no se podían curar, solo ser tratadas a lo largo del tiempo, lo que proporcionaba un patrón de síntomas. A pesar de que estas condiciones tuvieron un impacto importante en las actividades de la vida diaria, disminuyendo las acciones de autocuidado y aumentando la dependencia de un cuidador, la falta de información sobre las condiciones crónicas derivadas de su enfermedad original y su tratamiento desafió la familiaridad y la congruencia del evento. Sumado a la condición clínica como tal, es bien sabido que factores sociodemográficos como el género, nivel educativo y situación socioeconómica tienen un impacto tanto en el nivel de incertidumbre como en la manera en que esta se experimenta ${ }^{(22)}$, lo cual definitivamente debe ser tomado en cuenta en aras de brindar un cuidado integral.

La teoría también establece que la capacidad cognitiva 
Tabla 1. Plan de Cuidados para el Sr. R.

\begin{tabular}{|c|c|c|c|}
\hline Diagnóstico NANDA & NOC & NIC & Evaluación \\
\hline $\begin{array}{l}\text { Afrontamiento ineficaz } \\
\text { relacionado con incerti- } \\
\text { dumbre (00069) } \\
\text { Características definito- } \\
\text { rias } \\
\text { Empleo de formas de } \\
\text { afrontamiento que } \\
\text { impiden una conducta } \\
\text { adaptativa } \\
\text { Expresa incapacidad para } \\
\text { el afrontamiento }\end{array}$ & $\begin{array}{l}\text { Nivel de estrés } \\
\text { (1212) } \\
\text { Indicadores } \\
\text { 1) Irritabilidad, } \\
\text { 2) Depresión, } \\
\text { 3) Ansiedad }\end{array}$ & $\begin{array}{l}\text { Mejorar el afrontamiento (5230) } \\
\text { Actividades } \\
\text { Fomento de la verbalización de sentimientos, } \\
\text { percepciones y temores; y estimulación de la } \\
\text { participación familiar. } \\
\text { La inteligencia emocional y la expresión de la } \\
\text { emoción facilitan el comportamiento de adap- } \\
\text { tación al enfrentar el estrés, por lo tanto, el Sr. } \\
\text { R tuvo tiempo de hablar sobre sus sentimientos } \\
\text { y pensamientos acerca de sus condiciones y se } \\
\text { le escuchó y enfatizó la importancia del reco- } \\
\text { nocimiento de la emoción y la expresión de la } \\
\text { emoción. } \\
\text { Se alentó a la compañía de su esposa en esta } \\
\text { intervención para que el Sr. R pudiera fortalecer } \\
\text { la relación con ella y mejorar sus habilidades de } \\
\text { comunicación. }\end{array}$ & $\begin{array}{l}\text { Todos los indicadores del NOC } \\
\text { (Irritabilidad, Depresión y Ansie- } \\
\text { dad) mejoraron de moderado } \\
\text { (3) a leve (4). }\end{array}$ \\
\hline $\begin{array}{l}\text { Conocimientos defi- } \\
\text { cientes relacionado con } \\
\text { información insuficiente } \\
\text { (00126) }\end{array}$ & $\begin{array}{l}\text { Conocimiento: } \\
\text { manejo de la en- } \\
\text { fermedad crónica } \\
(1747) \\
\text { Indicadores } \\
\text { 1) Curso habitual } \\
\text { del proceso de la } \\
\text { enfermedad, } \\
\text { 2) Estrategias para } \\
\text { prevenir complica- } \\
\text { ciones } \\
\text { 3) Efectos tera- } \\
\text { péuticos de la } \\
\text { medicación } \\
\text { 4) Efectos adver- } \\
\text { sos de la medica- } \\
\text { ción. }\end{array}$ & $\begin{array}{l}\text { Enseñanza: proceso de enfermedad (5602) } \\
\text { Actividades } \\
\text { Se implementó una sesión educativa como parte } \\
\text { de las actividades; antes de la sesión el conoci- } \\
\text { miento acerca de sus condiciones de salud-enfer- } \\
\text { medad fueron explorados y reconocidos. } \\
\text { Luego, se habló con el Sr. R sobre el hipotiroidis- } \\
\text { mo y la ceguera (incluido su diagnóstico, fisio- } \\
\text { patología, signos y síntomas y complicaciones), } \\
\text { el tratamiento médico para todas sus afecciones } \\
\text { (incluidos los efectos secundarios y el tamaño de } \\
\text { la píldora, por lo que el Sr. R podría diferenciar } \\
\text { cada medicamento), información básica sobre la } \\
\text { movilización con equipo de ayuda (bastón) y la } \\
\text { importancia de los controles de salud constantes } \\
\text { que debe de hacerse en el centro de atención } \\
\text { primaria de salud. }\end{array}$ & $\begin{array}{l}\text { Los indicadores del NOC mejo- } \\
\text { raron en la siguiente manera: } \\
\text { Curso habitual del proceso } \\
\text { de la enfermedad mejoró de } \\
\text { conocimiento sustancial (4) a } \\
\text { conocimiento extenso (5) } \\
\text { Estrategias para prevenir } \\
\text { complicaciones mejoró de } \\
\text { conocimiento moderado (3) a } \\
\text { conocimiento extenso (5) } \\
\text { Efectos terapéuticos de la } \\
\text { medicación y Efectos adver- } \\
\text { sos de la medicación mejoró } \\
\text { de conocimiento escaso (2) a } \\
\text { conocimiento extenso (5). }\end{array}$ \\
\hline
\end{tabular}

Fuente: Elaboración propia basado en la Taxonomía II de la NANDA internacional, Nursing Intervention Classification (NIC) y Nursing Outcome Classification (NOC). 
y los proveedores de estructura influyen en el marco de estímulos que afectan directa o indirectamente la evaluación de la incertidumbre (1). A pesar de que el Sr. R estaba cognitivamente intacto, se podía notar una sobrecarga de información referente a las condiciones críticas en las que se había encontrado anteriormente: los proveedores de estructura se habían abocado a brindarle gran cantidad de información referente al macroadenoma, la meningitis y el defecto en el hueso esfenoides, sin abordar las condiciones crónicas con las que el Sr. R egresaría. Sin embargo, el apoyo social que recibió de su familia y su comunidad fue un factor protector. Por lo tanto, la experiencia de la enfermedad del Sr. R se interpretó como ambigua, compleja, impredecible y carente de información1. La incertidumbre del Sr. R se evaluó a través de la inferencia como un peligro, y su estrategia de afrontamiento en respuesta fue el control del afecto, debido a su constante evitación de situaciones estresantes. En consecuencia, los profesionales de enfermería requerían centrarse en el desarrollo o fortalecimiento de estrategias de afrontamiento emocional para lograr la adaptación y tener una nueva visión de la vida que integra la incertidumbre en su vida.

\section{CONCLUSIONES}

El proceso de enfermería requiere un marco teórico y / - conceptual para comprender mejor las necesidades humanas de los individuos y desarrollar intervenciones de calidad basadas en la evidencia. La identificación de una teoría desde los inicios del proceso de enfermería ayudó a interpretar, organizar y explicar los hallazgos de la valoración, lo cual guió a la identificación de los diagnósticos principales (afrontamiento ineficaz relacionado con incertidumbre y conocimientos deficientes relacionado con información insuficiente). Estos diagnósticos fueron intervenidos por medio de estrategias de afrontamiento y enseñanza para mejorar resultados relacionados con el nivel de estrés y conocimiento del manejo de la enfermedad crónica con el fin de ayudarle a la persona a adaptarse a la incertidumbre de su nueva situación de salud. En este caso, la teoría de la incertidumbre facilitó una atención más personalizada basada en evidencia científica. Además, el lenguaje estandarizado fue útil para cerrar la brecha entre la teoría y la práctica, facilitando al mismo tiempo la continuidad de la atención de enfermería entre los niveles de atención o los entornos.

\section{CONFLICTO DE INTERESES}

Los autores declaran no tener ningún conflicto de intereses.

\section{FINANCIAMIENTO}

Sin financiemiento.

\section{REFERENCIAS BIBLIOGRÁFICAS}

1. Mishel M. Uncertainty in illness. J Nurs Sch [Internet]. 1988 [citado el 16 de Mayo de 2018]; 20(4): 225-232. Disponible en: https://doi.org/10.1111/j.1547-5069.1988.tb00082.x

2. Mishel M. Reconceptualization of the uncertainty in illness theory. J Nurs Sch [Internet]. 1990 [citado 16 de Mayo de 2018]; 22: 256-262. Disponible en: https://doi. org/10.1111/j.1547-5069.1990.tb00225.x

3. Chibbaro S, Ganau M, Gubian A, Scibilia A, Todeschi J, Riehm S, Moliere S, Debry C, Goichot B, Proust F, Cebula $\mathrm{H}$. The Role of endoscopic endonasal approach in the multimodal management of giant pituitary adenoma: Case report and literature review. Asian J Neurosurg [Internet]. 2018 [citado 29 de Junio de 2019]; 13: 888-92. Disponible en: https://doi.org/10.4103/ajns.AJNS_97_18

4. Carosi G, Malchiodi E, Ferrante E, Sala E, Verrua E, Profka E, Giavoli C, Filopanti M, Beck-Peccoz P, Spada A, Mantovani G. Hypothalamic-Pituitary Axis in non-functioning pituitary adenomas: Focus on the prevalence of isolated central hypoadrenalism. Neuroendocrinology [Internet]. 2015 [citado 29 de Junio de 2019]; 102: 267-73. Disponible en: https://doi. org/10.1159/000430815

5. Simpson J, Heath J, Wall G. Living with a pituitary tumour: A narrative analysis. Psychol Health [Internet]. 2014 [citado 29 de Junio de 2019]; 29(2): 162-76. Disponible en: http:// dx.doi. org/10.1080/08870446.2013.840784

6. Yang CJ, Huang GS, Xiao FR, Lou MF. Symptom distress and quality of life after stereotactic radiosurgery in patients with pituitary tumors: A questionnaire survey. PLOS ONE [Internet]. 2014 [citado 29 de Junio 2019]; 9(2): 1-7. Disponible en: http://dx.doi.org/10.1371/journal.pone.0088460

7. Wilkinson JM. Nursing process and critical thinking. New Jersey: Prentice Hall; 2012.

8. Gordon M. Manual de diagnósticos enfermeros. España: Elsevier; 2003.

9. Herdman $T$, Kamitsuru $S$, editors. NANDA International nursing diagnoses: definitions and classification, 2015-2017. Oxford: Wiley-Blackwell; 2014.

10. Moorhead S, Johnson M, Mass M, Swanson, E, editors). Nursing Outcomes Classification (NOC). 5th ed. MO: Mosby; 2012.

11. Bulechek G, Butcher H, Dochterman J, Wagner C, editors. Nursing Interventions Classification (NIC). 6th ed. MO: Mosby; 2012. 
12. Escuela de Enfermería. Malla curricular del Plan de Estudio de Licenciatura en Enfermería. Universidad de Costa Rica [Internet]. 2016 [citado 15 de Mayo de 2019]. Disponible en: Disponible en: http://enfermeria.ucr.ac.cr/images/plan\%20 de\%20estudios.pdf

13. Wuryanto E, Rahayu G, Emilia O, Harsono. Implementation and Evaluation of Outcome-Present Test (OPT) - Peer learning model to improve clinical reasoning skill among nursing students. Int J Sci Eng Res [Internet]. 2017 [citado 20 de Julio de 2018]; 8(5): 895-903. Disponible en: http://www. atmph.org/article.asp?issn=1755-6783; year $=2017$; volume $=1$ 0 ;issue $=3$; spage $=657$; epage $=663$; aulast $=$ Wuryanto

14. Kurita K, Garon EB, Stanton AL, Meyerowitz BE. Uncertainty and psychological adjustment in patients with lung cancer. Psycho-Oncology [Internet]. 2013 [citado 4 de Junio de 2018]; 22(6): 1396-401. Disponible en: https://doi. org/10.1002/pon.3155

15. Giammanco MD, Gitto L. Coping, uncertainty and health-related quality of life as determinants of anxiety and depression on a sample of hospitalized cardiac patients in Southern Italy. Qual Life Res [Internet]. 2016 [citado 4 de Junio de 2018]; 25(11): 2941-56. Disponible en https://doi. org/10.1007/ s11136-016-1323-5

16. Parker PA, Davis JW, Latini DM, Baum G, Wang X, Ward JF, Kuban D, Frank SJ, Lee AK, Logothetis CJ, Kim J. Relationship between illness uncertainty, anxiety, fear of progression and quality of life in men with favourable-risk prostate cancer undergoing active surveillance. BJU Int [Internet]. 2016 [citado 20 de Julio de 2018]; 117: 469-77. Disponible en https://doi.org/10.1111/ bju.13099

17. Mishel MH, Germino BB, Lin L, Pruthi RS, Wallen EM, Crandell J, Blyler D. Managing uncertainty about treatment decision making in early stage prostate cancer: a randomized clinical trial. Patient Educ Couns [Internet]. 2009 [citado 20 de Julio de 2018]; 77(3): 349-59. Disponible en https://doi. org/10.1016/j. pec.2009.09.009

18. Parker P, Alba F, Fellman B, Urbauer D, Li Y, Karam J, Tannir $N$, Jonasch E, Wood C, Matin S. Illness Uncertainty and Quality of life of patients with small renal tumors undergoing watchful waiting: A 2-year prospective study. European Urology [Internet]. 2013 [citado 12 de Agosto de 2018]; 63(6): 1122-1127. Disponible en: https://doi.org/10.1016/j. eururo.2013.01.034

19. Schiele SE, Emery CF, Jakcson JL. The role of illness uncertainty in the relationship between disease knowledge and patient-reported outcomes among adolescents and adults with congenital heart disease. Heart \& Lung [Internet]. 2018 [citado 12 de Agosto de 2018]; 00(2018): 1-6. Disponible en: https:// doi.org/10.1016/j.hrtlng.2018.10.026
20. Zhang Y. Uncertainty in illness: Theory review Application, and Extension. Oncol Nurs Forum [Internet]. 2017 [citado 16 de Mayo 2018]; 44(6): 645-649. Disponible en doi: http:// dx.doi. org/10.1188/17.ONF.645-649

21. Hurt CS, Cleanthous S, Newman SP. Further explorations of illness uncertainty: carers' experiences of Parkinson's disease. Psychology \& Health [Internet]. 2017 [citado 12 Agosto 2018]; 32(5): 549-66. Disponible en: https://doi.org/10.1080/088704 46.2017.1283041

22. Blake A, Asnani V, Leger RR, Harris J, Odesina V, Hemmings DL, Morris DA, Knight-Madden J, Wagner L y Asnani MR. Stigma and illness uncertainty: adding to the burden of sickle cell disease. Hematology [Internet]. 2018 [citado 7 Agosto 2018]; 23(2): 128-30. Disponible en: https:// doi.org/10.1080/10245332.2017.13598 98 